NASA-CR-203018

\title{
Rocket FUV Observations of the Io Plasma Torus During the Shoemaker-Levy/9 Impacts
}

\author{
S.A. Stern, ${ }^{1}$ D. Slater, ${ }^{2}$ W. Cash,${ }^{3}$ E. Wilkinson,${ }^{3}$ J. Green,${ }^{3} \&$ R. Gladstone ${ }^{2}$
}

Abstract. We observed the lo torus from 820-1140 $\AA$ on UT 20.25 July 1994 from a sounding rocket telescope/spectrograph. These observations serve as only the fourth published spectrum of the torus in this wavelength range, and the only FUV data documenting the state of the torus during the Shoemaker-Levy 9 impacts.

\section{Introduction}

At 05:54UT on 20 July 1994 our EUVS telescope and spectrograph payload was successfully launched on a Black Brandt IX sounding rocket from White Sands, New Mexico, to obtain far ultraviolet spectra of Jupiter and the Io plasma torus during the Shoemaker-Levy $/ 9$ (SL/9) impacts. The L impact had occured about 8 hours earlier and the $N$ impact was still over 4 hours in the future. We launched the payload at this time to satisfy a variety of launch window constraints and to obtain observations at a system III Central Meridian Longitude (CML) of $180 \pm 20$ degrees. CML 180 is the longitude at which the Jovian north polar UV aurora are historically observed to peak (Livengood et al. 1990). Concurrently, the Extreme Ultraviolet Explorer (EUVE) observatory was obtaining spectra of Jupiter and the torus from 70 to $760 \AA$, and the International Ultraviolet Explorer (IUE) was obtaining an 1150-1950 $\AA$ spectrum of the torus. The EUVS data essentially bridge the gap between the EUVE and the IUE spectra. The closest Rosat x-ray observatory observations began about 7 hours after the EUVS flight, approximately coincident with Hubble Space Telescope (HST) observations that included torus spectroscopy (cf., McGrath et al. 1995; Waite et al. 1995).

EUVS observed Jupiter from a mission elapsed time of $\mathrm{T}+115$ seconds (upleg, $162 \mathrm{~km}$ ) to $\mathrm{T}+390$ seconds (downleg, $193 \mathrm{~km}$ ). At apogee the zenith angles of Jupiter and the Sun were $78.4^{\circ}$ and $124^{\circ}$, respectively; the apparent Jupiter system III CML was $166^{\circ}$. The

\footnotetext{
'Southwest Research Institute, Boulder, Colorado

${ }^{2}$ Southwest Research Institute, San Antonio, Texas

${ }^{3}$ University of Colorado, Boulder, Culorarlo
}

Copyright 1995 by the American Geophysical Union.

Paper number 95GL01899

0094-8534/95/95GL-01899\$03.00
B0V flux calibration star Spica ( $\alpha$ Vir) was observed on the trajectory's downleg from $T+395 \sec (189 \mathrm{~km})$ to $\mathrm{T}+450 \mathrm{sec}(108 \mathrm{~km})$. Following the Spica observations, payload operations were halted. The payload has since been successfully reflown to obtain an EUV/FUV spectrum of Venus in August 1994, and the lunar atmosphere occultation of Spica in April 1995.

\section{Payload and Experiment Description}

The EUVS payload consists of an EUV telescope, an associated spectrograpl, and accompanying detector, power system, and telemetry electronics. The telescope is a diamond-turned f/15 Wolter type II grazing incidence design, with a $30 \mathrm{~cm}$ aperture (cf., Cash et al. 1989). The primary mirror is Ni coated; the secondary is $\mathrm{SiC}$ coated. Light from the telescope is focused onto the entrance slit. The EUVS spectrograph is a $0.4 \mathrm{~m}$, normal-incidence Rowland circle design. Together, the spectrograph and its grating produce a nominal plate scale of $13.9 \AA \mathrm{mm}^{-1}$. The spectrum is measured using a 2-D resistive-anode (Ranicon) detector with an active area measuring $25 \mathrm{~mm}$ in diameter; the Ranicon's microchannel plate is coated with a $\mathrm{KBr}$ photocathode sensitive in the $700-1500 \AA$ region of the ultraviolet. Because the detector is windowless, the 404 pound instrument is launched under vacuum.

For the Jupiter/SL9 flight we constructed a spectrograph entrance slit that measured 380" (spatial dimension) $\times 100$ " (dispersion dimension). Since we did not know, a priori, whether the SL/9 impacts would increase or decrease the emissions from Jupiter and the Io torus, the 100 " dispersion-dimension slit size was chosen to maximize the total counts measured during the flight by allowing all of the telescope's 40 " FWHM point spread function (PSF) to enter the slit. This sacrificed spectral resolution for counting statistics, but ensured that the entirety of the lo torus was imaged onto the detector. Because the entrance slit was larger than both Jupiter and the torus, the spectral resolution of the data was set by a combination of the instrument PSF and the size of the emitting regions, which nominally corresponds to $27.7 \AA$ for the torus observations. This is comparable to that obtained by the Voyager 1 and 2 UV spectrometer data (Broadfoot et al. 1979). The torus spectra made by EUVE. IUE, and ASTRO Hopkins Ultraviolet Trescope (IIIT) have resolutions of $\approx 3 A, \approx 11 A$, and $\approx 3 \Lambda$, respertively. Whereas EUVS, EUVE (Hall of al. I(9)4), and Voyager view the 
entire torus, HUT (Moos et al. 1991) and IUE observe only a portion of a single ansa in any given observation.

We centered EUVS' entrance slit on Jupiter, and oriented it to place its long axis parallel to Jupiter's equator. The 380 " slit length slit corresponded to $20.7 \mathrm{R}_{J}$ during the observations. As such. Jupiter and both torus ansae were simultaneously observed.

\section{Data and Reductions}

Figure 1 depicts the 2-D Jupiter/Io torus count spectrum EUVS obtained between $\mathrm{T}+146$ and $\mathrm{T}+370$ seconds, when the payload was above detectable telluric absorption. To cleanly separate the torus data from the Jupiter data, the 2-D detector image was divided into three regions: Io west ansa, Jupiter, and lo east ansa. The two Io torus regions were extracted as follows: First, we summed the entire Jupiter+lo torus spectrum over all wavelengths to create an average spatial profile. We then used a gaussian to fit the width of the Jupiter signal, and subtracted a normalized gaussian of this width and centroid from the data at each wavelength. Tests of the quality of this fit and subtraction of the Jupiter signal found there was $<3 \%$ Jovian contamination at most wavelengths, and $<6 \%$ contamination at any wavelength. In what follows we describe and then analyze the spectra of the east (dawn) and west (dusk) Io torus ansa. In a subsequent report we will analyze the EUVS spectrum of Jupiter itself.

The spectra were reduced as follows. First, the detector background was determined by least-squares fitting a straight line across the detector in each of 64 spatial bins along the length of the entrance slit. Only the region of the detector not exposed to light (i.e., the nonslit region) was used. The resulting fit in each spatial

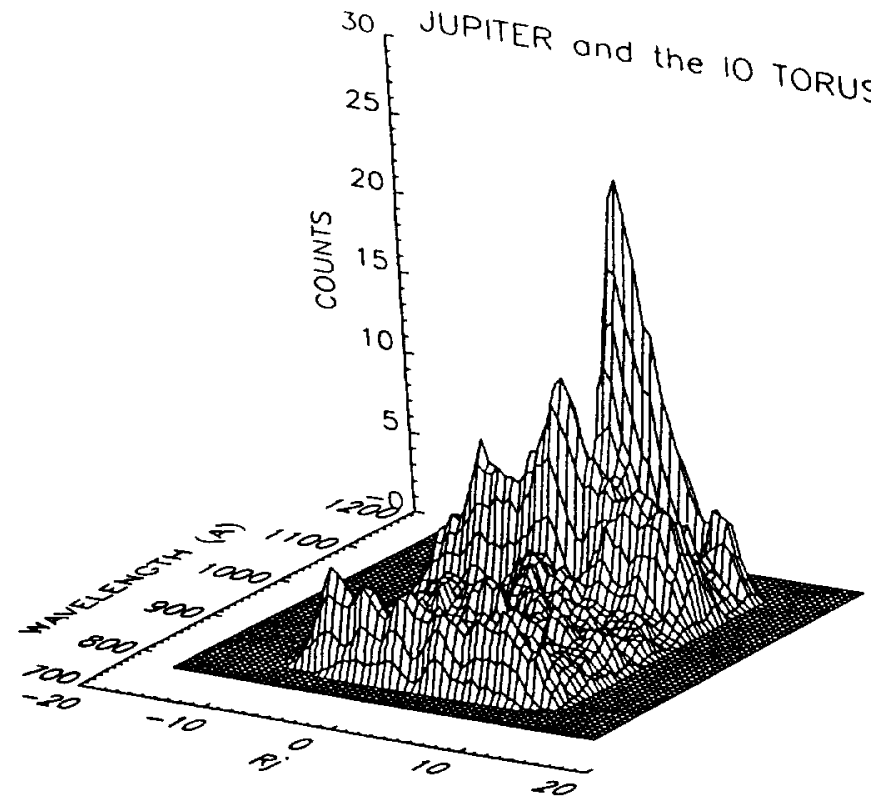

Figure 1. The background-removed Jupiter-system count spectrum obtained by EUVS. Emission from the two torus ansa and Jupiter are each clearly distinguishable; notice the brightness esymmetry between the two ansa (dusk, left; dawn right).
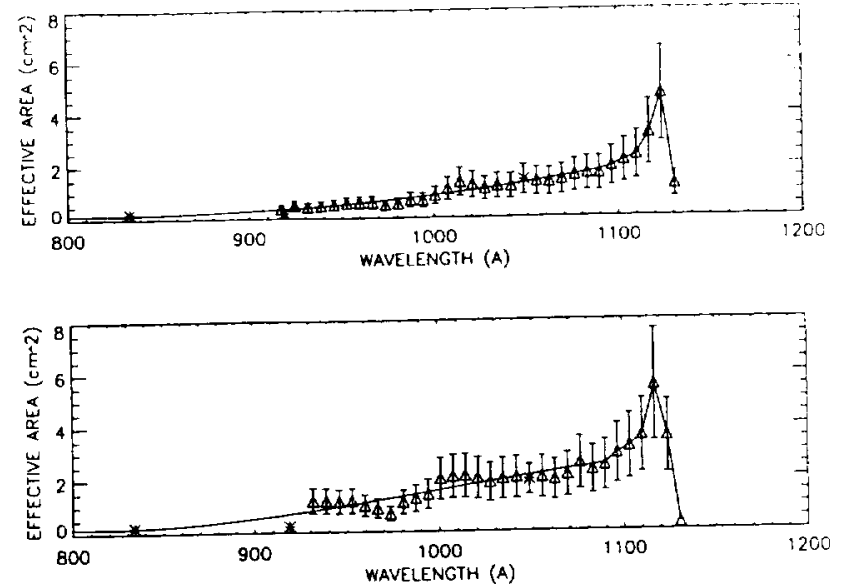

Figure 2. The effective area of the EUVS instrument derived from laboratory measurements (asterisks) and in-flight observations of the BOV star Spica (triangles). The error bars not shown are smaller than the asterisks. Top panel; west (dawn) region effective area; lower panel: east (dusk) region effective area.

bin was then used to estimate the detector barkground in the slit for that bin. For all bins the detector background was found to be $<1 \%$ of the signal in the slit. region of the detector. An equivalent background subtraction was performed on the Spica data.

Using the background-subtracted Spica clataset (with a total of 43,911 counts above the region of detectable telluric absorption), we computed separate effective area calibrations for the west and east torus ansa regions of the instrument. These are shown in Figures $2 \mathrm{a}$ and 2 b respectively. The effective area above $912 \AA$ was calculated using the Spica count rate spectrum and an archival flux reference spectrum of Spica (Holberg et al. 1982). Below 912 A, H ionization in the interstellar mediun introduces an opacily which cuts ofl the Spica specterum: therefore the only effertive area diata we have below $912 \AA$ are laboratory measurements obtained using an $\mathrm{O}^{+} / \mathrm{Ar}$ resonance source at $834 \AA$. The data points obtained from Spica are shown in Figure 2 as open triangles; data points obtained in the laboratory using calibrated oxygen and argon emissions are shown as asterisks. The spline-fit we adopted to the combined laboratory/Spica data are shown as a solid line in each panel. Using this fit, we converted the Io torus count rate spectra to flux spectra.

A wavelength scale was established by a least-squares fit to a series of Pt-lamp lines imaged onto the detector before and after the flight. These wavelength scales agree to within a few $\AA$ with the position of the $912 \AA$ ISM H opacity cutoff in the Spica spectrum we obtained in flight. The post-flight check of the wavelength scale detected a slight shift, of $2.4 \AA$ to the blue. This is an order of magnitude smaller than our spectral resolution, and does not affect the following analysis.

The flux rate spectra were converted to Rayleighs per $13.84 \AA$ spectral bin using the effective area of the instrument, the solid area of the slit, and slit filling factors derived by M. Taylor from our data. In order to provide 


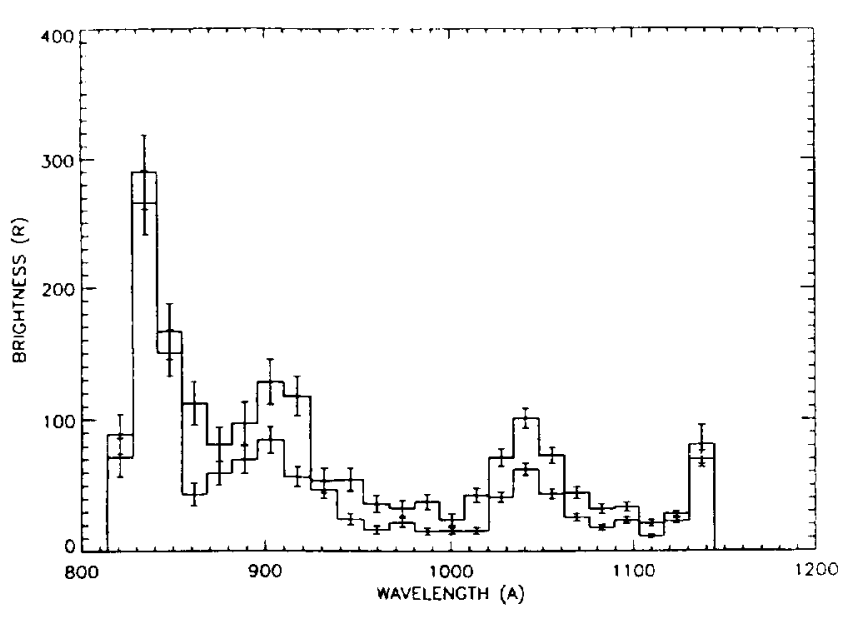

Figure 3. EUVS dawn (thin line) and dusk (thick line-spectra in brightness per $13.84 \mathrm{~A}$ bin. We used a slit filling factor of $7.3 \mathrm{for}$ $\lambda<903 \mathrm{~A}$ and 10.7 for $\lambda>903$. The main features are the 834 A OII/III blend, the $910 \mathrm{~A}$ SII blend, and the spectrally unresolved 1046 A SII complex.

Nyquist sampling, the wavelength binning was chosen to be twice the EUVS spectral resolution.

Figure 3 shows the brightness spectra we obtained for both the dawn and dusk ansa of the torus. Notice that because the EUVS instrument's effective area is much higher at the red end of the bandpass than at the blue end, these fluxes and brightnesses are quite different from the counts spectra shown in Figure 1.

Our radiative transfer calculations to predict telluric airglow spectra along the EUVS line of sight at the time of these observations indicates that no telluric emission exceeded $10 \mathrm{R}$, including $H \mathrm{I}$ Lyman $\beta$. As such, we conclude that the spectra shown here are not significantly contaminated by terrestrial emissions. We ascribe the following identifications to be the dominant source of the brightest three torus features in these spectra: 834 $\AA$ (O II/III), $910 \AA$ (S II), and $1046 \AA$ (SII). The weaker features have not yct been conclusively identified. Table 1 presents the derived brightnesses of the three bright torus emission features in tabular form, along with the measured fluxes and derived total radiated power of each ansa for each of these features. Each line brightness was oblained by integrating over the spectral resolution elements centered on the feature. The error bars are the rool-sum-square of the errors due to comnting statistics, olscrving geometry, detector background, and residual Jovian continmination sources of error. Fffective area calibration mucertainties create an additional merentainty in the absolute scale of 30 -
$40 \%$. We comment on three significant aspects of these data:

- Dusk/Daun Emission Asymmetries: The SL/9-epoch dusk/dawn ratio of the $834 \AA$ OII feature is $1.0 \pm 0.3$; the $910 \AA$ dusk/dawn SII ratio is $1.6 \pm 0.2$; the $1046 \AA$ SII ratio is $1.7 \pm 0.2$ (these error bars were computed by combining the uncertainties in our counting statistics, observing geometry, detector foreground, and Jovian contamination). The EUVS-derived sulfur asymmetry ratio compares relatively well with Voyager-derived values of 1.1-1.6 (Shemansky \& Sandel 1982). However, an 80,000 second EUVE lo torus observation in early 1993 revealed dusk/dawn emission asymmetry ratios of $2.7 \pm 0.7$ (OII $539 \AA$ ) and $2.3 \pm 0.3$ SIII $(685 \AA$ ), respectively (Hall et al. 1994). An EUVE SL/9-epoch dusk/dawn asymmetry was not reported in McGrath et al. (1995), but one of us who is involved in the EUVE SL/9-epoch data analysis (GRG) finds no significant difference from the the Hall et al. (1994) result. It appears the EUVS dusk/dawn asymmetry is significantly different from the EUVE asymmetry measurements, but is similar to the Voyager results.

- SII $(910 \AA) / O H(834 \AA)$ and SII $(1046 \AA) / O H I(834$ A) Line Ratios: Direct comparison of the brightnesses and fluxes of our data to Voyager 1 and 2 and HUT data are not easily made, since the observing geometries, resolutions, and slit filling factors of each observation are considerably different. Instead, we prefer to present sulfur ion/oxygen ion line ratios for the dawn, dusk, and dawn+dusk average spectra obtained by the various instruments. The advantage of this approach is that observing geometry and instrumental differences largely cancel. Table 2 presents these comparisons for the SII $(910 \AA) /$ OII $(8.34 \AA)$ and SII $(1046 \AA) / O I I(834$ $\AA)$ line ratios. The Voyager data source is Shemansky (1987); the HUT data sources are Moos et. al. (1991) and a personal communication (D.T. Hall 1995). Notice that the EUVS $\AA / 834 \AA$ line ratios are more like the 1979 Voyager 1 and 2 observations than the 1990 HUT observations. Now consider the SII (1046 A)/OII $(834 \AA)$ line ratios. Table 2 shows that the EUVS line ratio is about twice the IIUT line ratio. Owing to Voyager's spectral resolution limitations, these ratios are not available in the Voyager dataset.

- The Lack of strong sill+sill 1014-1021 A Feratures: This is in sharp conlrast to the IIUT and Voyager

Table 1. Major Io Plasma Torus Emissions (820-1140 $\AA$ ): 20.25 July 1994

\begin{tabular}{|c|c|c|c|}
\hline Parameter & $834 \AA(\mathrm{O} I \mathrm{I} / \mathrm{O} \mathrm{III})$ & $910 \AA(\mathrm{S} \mathrm{II})$ & $1046 \AA(S$ II $)$ \\
\hline Dusk Ansa Brightness ( $R$ ) & $527 \pm 85$ & $342 \pm 68$ & $245 \pm 40$ \\
\hline Dawn Ansa Brightness (R) & $505 \pm 81$ & $210 \pm 42$ & $147 \pm 24$ \\
\hline Dusk Ansa Flux (ergs $\left.\mathrm{cm}^{-2} \mathrm{~s}^{-1}\right)$ & $1.2 \pm 0.2 \times 10^{-10}$ & $5.1 \pm 1.0 \times 10^{-12}$ & $2.9 \pm 0.5 \times 10^{-11}$ \\
\hline Dawn Ansa Flux (ergs $\mathrm{cm}^{-2} \mathrm{~s}^{-1}$ ) & $1.2 \pm 0.2 \times 10^{-10}$ & $3.0 \pm 0.2 \times 10^{-12}$ & $1.9 \pm 0.3 \times 10^{-11}$ \\
\hline Dusk Ansa Power (ergs s ${ }^{-1}$ ) & $9.3 \pm 1.5 \times 10^{18}$ & $3.8 \pm 0.8 \times 10^{18}$ & $2.4 \pm 0.4 \times 10^{18}$ \\
\hline Dawn Ansa Power (ergs s $\left.{ }^{-1}\right)$ & $8.9 \pm 1.4 \times 10^{18}$ & $2.3 \pm 0.5 \times 10^{18}$ & $1.4 \pm 0.2 \times 10^{18}$ \\
\hline
\end{tabular}


Table 2. Comparative Torus Line Emission Ratios

\begin{tabular}{ccc}
\hline EUVS (1994.6) Dawn Ansa & 0.42 & 0.29 \\
EUVS (1994.6) Dusk Ansa & 0.65 & 0.47 \\
HUT 1 (1990.9) Dawn Ansa & 0.26 & 0.17 \\
HUT 1 (1990.9) Dusk Ansa & 0.16 & $\mathrm{~N} / \mathrm{A}$ \\
Vgr 1 (1979.3) Dawn+Dusk Avg & 0.42 & $\mathrm{~N} / \mathrm{A}$ \\
Vgr 2 (1979.6) Dawn+Dusk Avg & 0.52 & $\mathrm{~N} / \mathrm{A}$ \\
HUT 1 (1990.9) Dawn+Dusk Avg & 0.21 & $\mathrm{~N} / \mathrm{A}$ \\
EUVS (1994.6) Dawn+Dusk Avg & 0.54 & 0.38 \\
\hline
\end{tabular}

datasets, where this foature is almost as prominant as the $834 \mathrm{~A}$ Oll feature. It is also in contrast to expecta-

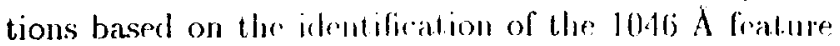
as SII (cf., the line strengths in Shemansky \& Smith 1981), and therefore warrants detailed analysis.

In conclusion, we have obtained the only SL/9-epoch Io plasma torus measurement in the important 820-1140 $\AA$ range. Comparisons to the three previously published EUV/FUV spectra of the Io torus reveal significant differences. Given the instrinsically-variable nature of the torus and the small number of torus observations in the EUV/FUV (which leave this wavelength region temporally-undersampled and therefore ill-constrained), it is not presently possible to determine $\mathrm{SL} / 9$ 's role in generating the spectrum we observed. We look forward to Galileo EUV/FUV studies, which should provide a much-expanded database from which to draw this judgement.

Acknowledgments. This paper is dedicated to the memory of the late John van Overeem at NASA/Wallops. We thank our NASA mission Manager Bob Spagnuolo, NASA Sounding Rocket Branch chief Ray Pless, Henry Brinton at NASA Headquarters, and the entire NASA/contractor team that made these time-critical observations a success. At SwRl, we thank project manager John Scherrer, and our field support staff, including Tom Booker, John McDonald, and Clarence McDonald. At CU we thank Scott McDonald.
We also thank our colleagues M. Táylor, N. Schneider, D. Shemansky, and D. Hall for useful conversations This work was supported by NAG5-5006.

\section{References}

Broadfoot, A.L., et al., 1979. Voyager 1 Observations of the Jupiter System Science, 204, 979.

Cash, W., et al. 1989. A Far-Ultraviolet Rocket-Borne Spectrograph. Exper. Space Sci., 1, 123-136.

Hall, D.T., et al., 1994. Extreme Ultraviolet Explorer Satellite Observations of Jupiter's Io Plasma Torus. A $p J$. Lett., 426, L51-54.

Livengood, T.A., and H.W. Moos, 1990. GRL, 17, 22652268.

Livengood, T.A., D.F. Strobel, and H.W. Moos, 1990. LongTerm Study of Longitudinal Dependence in Primary Particle Precipitation in the North Jovian Aurora, JGR, 95, 10375-10388.

McGrath, M.A., et al., 1995. Io Plasma Torus Response to Comet P/Shoemaker-Levy 9, Science, 267,1313-1317.

Moos, H.W., et al., 1991. Determination of Ionic Abundances in the Io Torus Using the Hopkins Ultraviolet Telescope. ApJ. Lett., 382, L105-110.

Shemansky, D.E., 1987. Ratio of oxygen to sulfur in the Io plasma torus. JGR, 92, 6141-6146.

Shemansky, D.E., and B.R. Sandel, 1982. The injection of energy into the lo plasma torus. JGR, 87, 219-229.

Waite, J.H., et al., 1995. ROSAT Observations of XRay Emissions from Jupiter during the Impact of Comet Shoemaker-Levy 9, Science, in press.

S.A. Stern, Southwest Research Institute, 1050 Walnut St., No. 429, Boulder, CO 80302

G.R. Gladstone and D.C. Slater, Southwest Research Institute, 6220 Culebra Rd., San Antonio, TX 78238

W.C. Cash, J.C. Green, and E. Wilkinson, CASA/CB391, University of Colorado, Boulder, CO 80309

(received December 15, 1994;

revised April 24, 1995;

accepted May 24, 1995.) 